\title{
Wedge diffraction in terms of the method of physical optics
}

\author{
Yusuf Z. Umul ${ }^{\text {a) }}$ \\ Electronic and Communication Dept., Cankaya University, \\ Balgat, Ankara 06530, Türkiye \\ a)yziya@cankaya.edu.tr
}

\begin{abstract}
The method of physical optics is extended for wedge diffraction. The classical integral of physical optics is taken into account for the diffraction problem of plane waves by a conducting halfplane. The integral is decomposed according to the transmitted and reflected scattered waves. The sinusoidal term in the integrand is rewritten by considering the fact that the half-plane is a special case of the wedge. Two cases of soft and hard surfaces are examined and the uniform diffracted waves are obtained by the asymptotic evaluation of the integrals. The results are compared with the literature numerically.
\end{abstract}

Keywords: physical optics, diffraction theory, wedge diffraction Classification: Electromagnetic theory

\section{References}

[1] H. M. Macdonald, "The effect produced by an obstacle on a train of electric waves," Phil. Trans. R. Soc. Lond. A, vol. 212, no. 1, pp. 299337, Jan. 1913.

[2] W. L. Stutzman and G. A. Thiele, Antenna Theory and Design, John Wiley \& Sons, New York, 1988.

[3] P. Ya. Ufimtsev, "Elementary edge waves and the physical theory of diffraction," Electromagn., vol. 11, no. 2, pp. 125-160, April 1991.

[4] G. L. James, Geometrical Theory of Diffraction for Electromagnetic Waves, IEE, London, 1983.

[5] K. Sakina, S. Cui, and M. Ando, "Mathematical derivation of modified edge representation for reduction of surface radiation integral," IEICE Trans. Electron., vol. E84-C, no. 1, pp. 74-83, Jan. 2001.

[6] Y. Z. Umul, "Modified theory of physical optics," Opt. Express, vol. 12, no. 20, pp. 4959-4972, Oct. 2004.

[7] Y. Z. Umul, "Modified theory of physical optics approach to wedge diffraction problems," Opt. Express, vol. 13, no. 1, pp. 216-224, Jan. 2005.

[8] Y. Z. Umul, "Modified theory of physical optics solution of impedance half plane problem," IEEE Trans. Antennas Propag., vol. 54, no. 7, pp. 2048-2053, July 2006.

[9] T. Shijo, L. Rodriguez, and M. Ando, "The modified-surface normal vectors in the physical optics," IEEE Trans. Antennas Propag., vol. 56, no. 12, pp. 3714-3722, Dec. 2008.

[10] N. Omaki, T. Shijo, and M. Ando, "PO with modified surface-normal 
vectors for RCS calculation of scatterers with edges and wedges," IEICE Trans. Electron., vol. E92-C, no. 1, pp. 33-39, Jan. 2009.

[11] Y. Z. Umul, "Effect of the shadow geometry on diffraction," J. Opt. Soc. Am. A, vol. 25, no. 12, pp. 2896-2900, Dec. 2008.

[12] G. Franceschetti, A. Iodice, A. Natale, and D. Riccio, "Stochastic theory of edge diffraction," IEEE Trans. Antennas Propag., vol. 56, no. 2, pp. 437-449, Feb. 2008.

[13] C. Bourlier and P. Pouliguen, "Useful analytical formulae for near-field monostatic radar cross section under the physical optics: Far-field criterion," IEEE Trans. Antennas Propag., vol. 57, no. 1, pp. 205-214, Jan. 2009.

[14] Y. Z. Umul, "The relation between the boundary diffraction wave theory and physical optics," Opt. Commun., vol. 281, no. 19, pp. 4844-4848, Oct. 2008.

[15] C. A. Balanis, Advanced Electromagnetic Engineering, Wiley, New York, 1989.

\section{Introduction}

The physical optics (PO) is a high frequency technique, which considers the radiation of induced surface currents on an equivalent plane at the point of scattering [1]. The method has been benefited in many areas of the electromagnetic theory. PO has some defects that limit the usage of the technique. The edge point contributions of the PO integrals lead to the incorrect edge diffracted waves [2]. There are some attempts in the literature in order to eliminate this drawback. For example some authors proposed to add or multiply correction factors, obtained from the exact solutions of the canonical diffraction problems $[3,4]$. Ando et al studied on the modification of the edge contour for the line integral reduction of the surface integrals [5]. Umul eliminated the mentioned defect of $\mathrm{PO}$ in its mathematical structure by defining three axioms based on the diffraction theory [6]. The asymptotic evaluation of the modified theory of physical optics (MTPO) integrals yielded the exact diffracted waves. Later he developed the method for wedges and impedance surfaces $[7,8]$. Ando et al studied on the same problem by defining modified surface normal vectors $[9,10]$. Another defect of PO is the zero current density in the shadow zone of the scatterer. For this reason PO represents a wedge diffraction problem with only one illuminated face, as a half-plane problem. Umul investigated this problem in terms of the construction of the MTPO integrals for the case of a conducting half-plane and showed that it is possible to express the shadow currents in terms of PO integrals [11].

It is the aim of this study to obtain a PO type integral for the scattered fields by a wedge. This approach may be seem to be trivial after the invention of the wedge diffraction integrals of MTPO [7], but the classical technique of $\mathrm{PO}$ is widely used at present by some authors in spite of its erroneous edge point contributions $[8,9]$. A second important point is the mathematical structure of the PO integrals. The transformation of the integrand of edge diffraction to the more general case of the wedge diffraction will give more 
insight about the construction of the method. The integrand will be decomposed according to the transmitted and reflected waves in order to derive the wedge diffracted waves. The obtained integrals will be evaluated asymptotically and the resultant fields will be compared with the wedge diffraction fields of PO and the exact theory.

A time factor of $\exp (j w t)$ is suppressed throughout the paper.

\section{Theory}

A conducting half plane, which is located at $y=0, x \in(0, \infty)$ and $z \in$ $(-\infty, \infty)$ is taken into account. An incident plane wave of $u_{0} \exp \left[j k\left(x \cos \phi_{0}+\right.\right.$ $\left.\left.y \sin \phi_{0}\right)\right]$ is illuminating the screen. $u_{0}$ is the complex amplitude of the electric or magnetic field. $\phi_{0}$ is the angle of incidence. $k$ is the wave-number. Two boundary conditions are considered as the conditions of Dirichlet (total field is equal to zero on the soft surface) and Neumann (the normal derivative of the total field is equal to zero on the hard surface). The total scattered waves can be written as

$$
u_{t}(P)=u_{i}(P)-\frac{k u_{0} \sin \phi_{0} \exp (j \pi / 4)}{\sqrt{2 \pi}} \int_{0}^{\infty} \exp \left(j k x^{\prime} \cos \phi_{0}\right) \frac{\exp (-j k R)}{\sqrt{k R}} d x^{\prime}
$$

and

$$
u_{t}(P)=u_{i}(P)+\frac{k u_{0} \exp (j \pi / 4)}{\sqrt{2 \pi}} \int_{0}^{\infty} \sin \beta \exp \left(j k x^{\prime} \cos \phi_{0}\right) \frac{\exp (-j k R)}{\sqrt{k R}} d x^{\prime}
$$

for soft and hard surfaces respectively [14]. $u_{i}(P)$ is the incident field at the observation point. $R$ is the distance between the integration and observation points. $\beta$ is the angle between the $-x$ axis and $R$. We will multiply and divide the integrands of Eqs. (1) and (2) by $\cos \phi_{0}-\cos \beta$. The trigonometric relations of

$$
\frac{1}{\cos \phi_{0}-\cos \beta}=\frac{1}{2 \sin \phi_{0}}\left(\cot g \frac{\beta-\phi_{0}}{2}-\cot g \frac{\beta+\phi_{0}}{2}\right)
$$

and

$$
\frac{1}{\cos \phi_{0}-\cos \beta}=\frac{1}{2 \sin \beta}\left(\cot g \frac{\beta-\phi_{0}}{2}+\cot g \frac{\beta+\phi_{0}}{2}\right)
$$

can be obtained for the soft and hard surfaces respectively. Thus the PO integrals can be expressed as

$$
\begin{aligned}
& u_{P O 1}(P) \\
& =\frac{k u_{0} \exp (j \pi / 4)}{2 \sqrt{2 \pi}} \\
& \quad \times \int_{0}^{\infty} \exp \left(j k x^{\prime} \cos \phi_{0}\right)\left(\cos \phi_{0}-\cos \beta\right) \cot g \frac{\beta-\phi_{0}}{2} \frac{\exp (-j k R)}{\sqrt{k R}} d x^{\prime}
\end{aligned}
$$

and 


$$
\begin{aligned}
& u_{\mathrm{PO} 2}(P) \\
& =\frac{k u_{0} \exp (j \pi / 4)}{2 \sqrt{2 \pi}} \\
& \quad \times \int_{0}^{\infty} \exp \left(j k x^{\prime} \cos \phi_{0}\right)\left(\cos \phi_{0}-\cos \beta\right) \cot g \frac{\beta+\phi_{0}}{2} \frac{\exp (-j k R)}{\sqrt{k R}} d x^{\prime}
\end{aligned}
$$

for the soft surface. The total scattered field is equal to $u_{i}(P)-u_{P O 1}(P)+$ $u_{P O 2}(P)$. We will deal with the soft surface. The same operations are also valid for the hard surface. The cotangent functions, in the integrands of Eqs. (5) and (6), can be written as

$$
\cot g \frac{\beta \mp \phi_{0}}{2}=\tan \frac{\pi-\left(\beta \mp \phi_{0}\right)}{2} .
$$

Now we take into account the wedge diffraction geometry in Fig. 1.

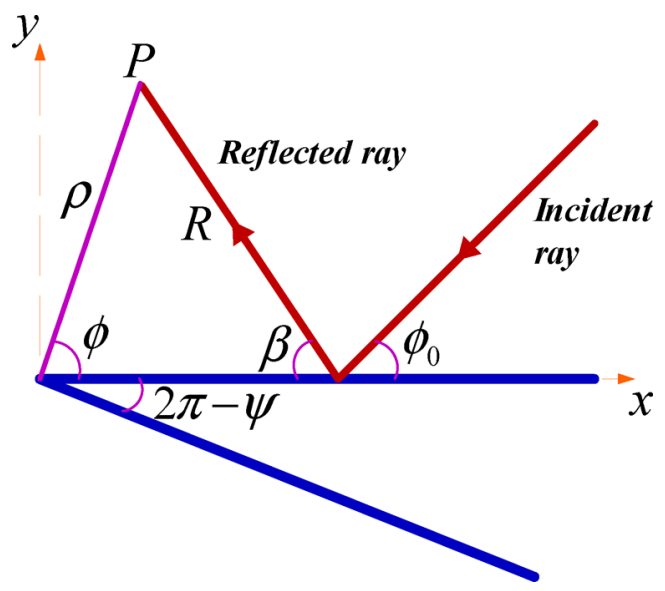

Fig. 1. Geometry of the wedge

$\psi$ is the outer angle of the wedge. The parameter of $n$ is defined as $\psi / \pi$. It is apparent that $n$ is equal to 2 for a half-plane, because the outer angle of a half-plane is $2 \pi$. With this knowledge in mind, Equation (7) can be rewritten as

$$
\cot g \frac{\beta \mp \phi_{0}}{2}=\left.\sin (\pi / n) \frac{\cos (\pi / n)-\sin \left\{\left[\pi-\left(\beta \mp \phi_{0}\right)\right] / n\right\}}{\cos (\pi / n)-\cos \left\{\left[\pi-\left(\beta \mp \phi_{0}\right)\right] / n\right\}}\right|_{n=2}
$$

for wedge diffraction. Note that Eq. (8) directly reduces to Eq. (7) for $n=2$. As a result the PO integrals can be obtained as

$$
u_{P O 1,2}(P)=\frac{k u_{0} \exp (j \pi / 4)}{n \sqrt{2 \pi}} \int_{0}^{\infty} \exp \left(j k x^{\prime} \cos \phi_{0}\right) f_{\mp} \frac{\exp (-j k R)}{\sqrt{k R}} d x^{\prime}
$$

where $f_{ \pm}$is equal to Eq. (8) multiplied by $\cos \phi_{0}-\cos \beta$. The signs of - and + are related with the subscripts of 1 and 2, respectively. Equation (9) enables one to evaluate the wedge diffracted waves with the PO integration. When compared with Ref. [7], which introduced the MTPO integrals that lead to the exact wedge diffracted waves, it can be seen that the only difference occurs from the numerator of Eq. (10). This is the same term that causes the erroneous edge diffracted fields of PO [14]. 


\section{Asymptotic evaluation of the PO integral}

In this section we will introduce the wedge diffracted fields of PO by the uniform asymptotic evaluation of Eq. (9). The details of the method can be found in Refs. $[6,7,8]$. At the edge point, $\beta$ and $R$ are equal to $\pi-\phi$ and $\rho$, respectively. The uniform wedge diffracted waves of PO can be written as

$$
u_{P O}=h_{-} \operatorname{sign}\left(\xi_{-}\right) F\left[\left|\xi_{-}\right|\right]-h_{+} \operatorname{sign}\left(\xi_{+}\right) F\left[\left|\xi_{+}\right|\right]
$$

for $h_{ \pm}$can be expressed as

$$
\begin{aligned}
h_{\mp}= & \frac{u_{0} 2 \sin (\pi / n)}{n} \exp \left[j k \rho \cos \left(\phi \mp \phi_{0}\right)\right] \cos \frac{\phi \mp \phi_{0}}{2} \\
& \times \frac{\cos (\pi / n)-\sin \left\{\left(\phi \mp \phi_{0}\right) / n\right\}}{\cos (\pi / n)-\cos \left\{\left(\phi \mp \phi_{0}\right) / n\right\}}
\end{aligned}
$$

according to Eq. (8). $\operatorname{sign}(x)$ is the signum function, which is equal to one for $x>0$ and -1 otherwise. $F[x]$ is the Fresnel function that can be defined as

$$
F[x]=\frac{\exp (j \pi / 4)}{\sqrt{\pi}} \int_{x}^{\infty} \exp \left(-j t^{2}\right) d t .
$$

$\xi_{ \pm}$is the detour parameter and can be given by the equation of

$$
\xi_{ \pm}=-\sqrt{2 k \rho} \cos \frac{\phi \pm \phi_{0}}{2} .
$$

Since the upper face of the wedge is illuminated, the surface current that will flow on the surface at $\phi=\psi$ is equal to zero according to the method of PO. The PO integral is evaluated for the face at $\phi=0$ and is equal to Eq. (1). Thus the scattered fields of PO by a wedge can be obtained as the special case of Eq. (10) for $n=2$. The uniform expression of the scattered fields can be written as

$$
u_{P O c}=\left.h_{-}\right|_{n=2} \operatorname{sign}\left(\xi_{-}\right) F\left[\left|\xi_{-}\right|\right]-\left.h_{+}\right|_{n=2} \operatorname{sign}\left(\xi_{+}\right) F\left[\left|\xi_{+}\right|\right]
$$

immediately for the classical PO. The exact diffracted fields can be evaluated by subtracting the GO waves from the exact series solution, given in Ref. [15]. The GO fields can be written as

$$
u_{G O}=u_{0}\left\{\exp \left[j k \rho \cos \left(\phi-\phi_{0}\right)\right] U\left(-\xi_{-}\right)-\exp \left[j k \rho \cos \left(\phi+\phi_{0}\right)\right] U\left(-\xi_{+}\right)\right\}
$$

where $U(x)$ is the unit step function, which is equal to one for $x>0$ and zero otherwise.

\section{Numerical analysis}

In this section, we will compare the uniform diffracted fields by wedge for the extended PO, classical PO and exact solution.

Figure 2 shows the variation of the diffracted fields of exact, MTPO, extended PO and classical PO methods, with respect to the observation angle. The diffracted waves of MTPO can be obtained by eliminating the terms in the numerator of Eq. (8) [7]. The distance of observation $(\rho)$ is 


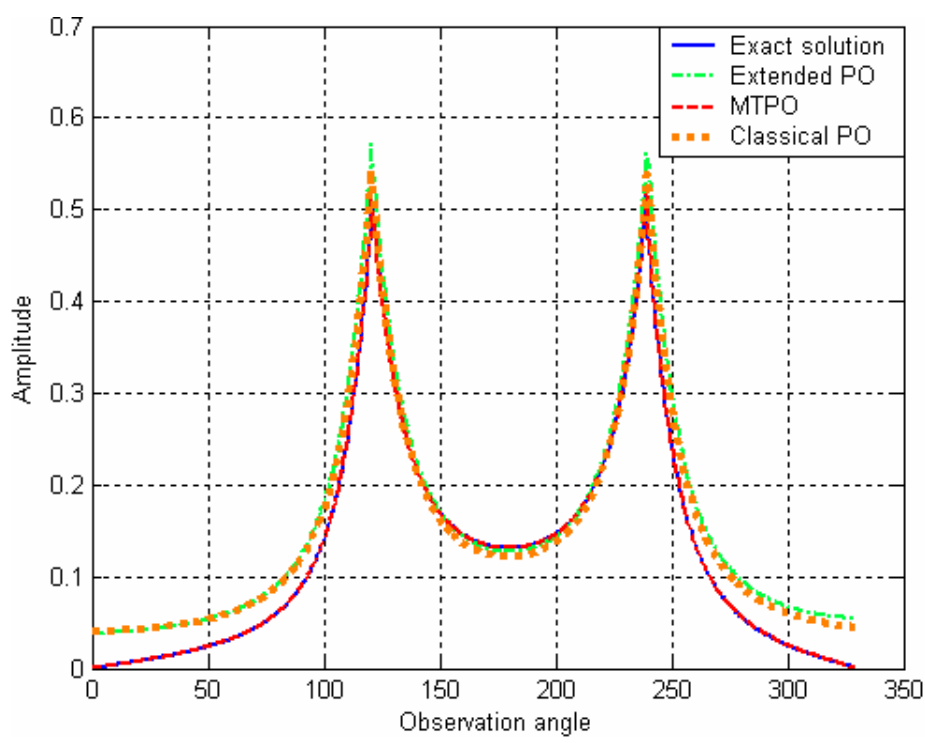

Fig. 2. Comparison of the diffracted waves

taken as $6 \lambda$ where $\lambda$ is the wavelength. The angle of incidence $\left(\phi_{0}\right)$ is equal to $60^{\circ}$. The outer angle of the wedge $(\psi)$ is $330^{\circ}$. It can be seen that the field that best fits to the exact solution is the diffracted waves of MTPO. The extended PO approaches to the exact solution between the angles of $110^{0}$ and $250^{\circ}$. But the classical and extended PO does not satisfy the boundary conditions.

\section{Conclusion}

In this study, we extended the classical method of PO for the problem of diffraction of plane waves by a conducting wedge. The obtained integral, in Eq. (9), gives information about the transition between the classical PO and MTPO. The numerical results show that the extended PO is also does not in exact harmony with the exact solution. This behavior is the result of the term in the numerator of Eq. (8), which is also the reason of the erroneous diffracted waves of classical PO. When these terms are eliminated, the scattered waves of MTPO are obtained. Figure 2 shows that these improved field expressions are in exact harmony with the rigorous solution. 